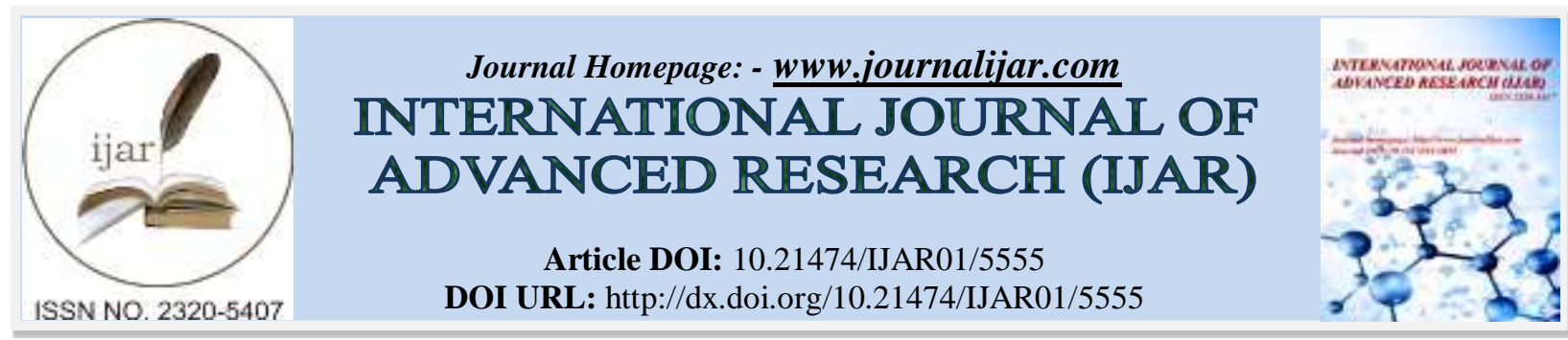

RESEARCH ARTICLE

\title{
HAND HYGIENE KNOWLEDGE AND PRACTICE AMONG MEDICAL STUDENTS IN KING ABDULAZIZ UNIVERSITY HOSPITAL.
}

\author{
Salma H. Almadani ${ }^{1}$, Yara A. Kano ${ }^{1}$, Lama A. Rayyis ${ }^{1}$, Weaam S. Magram ${ }^{1}$, Alaa A. Ismail ${ }^{1}$ and Dr. Eman \\ K. Aldigs ${ }^{2}$. \\ 1. College of Medicine, King Abdulaziz University, Jeddah, Kingdom of Saudi Arabia. \\ 2. Assistant Professor, Department of Medical Microbiology, King Abdulaziz University, Jeddah, Kingdom of \\ Saudi Arabia.
}

\section{Manuscript Info}

(n.........................

Manuscript History

Received: 06 August 2017

Final Accepted: 08 September 2017

Published: October 2017

Key words:-

Hand hygiene, Nosocomial infections,

Hospital acquired infections.

\section{Abstract}

Background: Hand hygiene $(\mathrm{HH})$ is considered the most beneficial method to reduce the incidence of Hospital-Acquired Infections. However, compliance to $\mathrm{HH}$ by healthcare workers are reportedly poor with a compliance rate of $40 \%$. In spite of the reports concerning the knowledge of $\mathrm{HH}$ among medical students in many countries, Saudi Arabia has greatly lagged behind in this aspect.

Aim: This study aims to evaluate HH knowledge and practice among first and last clinical years; (i.e. fourth and sixth year respectively) at King Abdulaziz University Hospital (KAUH), Jeddah, Saudi Arabia.

Methods: A cross-sectional study was conducted on 261 medical students of fourth and sixth year, at KAUH in Jeddah, Saudi Arabia. A covert observation was used to assess the practice and a 5-point Likert scale questionnaire was used for evaluating the students' knowledge. Both were based on the WHO five moments of $\mathrm{HH}$. A consent was obtained from all participants. Data analysis was done using SPSS version 21 and Pearson's Chi-Square was used for association.

Results: Medical students included in the study were fourth and sixth year students accounting as $157(60 \%)$, and $104(40 \%)$ respectively. Practice among sixth year students was found to be better than fourth year students $(p=0.004)$. As for the knowledge between fourth year and sixth year students it was found to be similar and markedly high ( $\mathrm{p}$ $=0.5$ ), ranging between strongly agree and agree in the questionnaire.

Conclusion: Strict protocols are needed for the implementation of $\mathrm{HH}$ practice among students.

Copy Right, IJAR, 2017,. All rights reserved.

\section{Introduction:-}

Hospital-acquired infections (HAIs) otherwise called nosocomial infections are one of the most common preventable medical complications due to unsafe clinical practice ${ }^{1-4}$ They increase the length of hospital stay, morbidity and mortality and are also associated with higher microbial drug resistance. In addition, HAIs impose a huge burden on healthcare systems ${ }^{1,2,5,6}$. Over 1.4 million people around the world have been affected by HAIs. It was estimated that developing countries have a two to 20 times more increased risk of healthcare related infections in comparison with that of the developed countries ${ }^{7,8}$ 
Healthcare workers' hands continuously become colonized with commensal flora which are replaced by pathogenic organisms that cause transmission of nosocomial infections ${ }^{9-11}$. Thus, hand hygiene $(\mathrm{HH})$ is considered the single most beneficial and cost-effective method to decrease the incidence of HAIs ${ }^{2,12-14}$. However, compliance to HH guidelines by healthcare workers are reportedly poor with an average compliance of $40 \%^{5,9,12,14-16}$.

Studies showed that healthcare workers' compliance to HH is generally low ${ }^{4,5}$. Twenty-three percent of clinicians and medical students adhere to $\mathrm{HH}$ practice ${ }^{17}$. Another study conducted among medical students have shown that their average awareness of $\mathrm{HH}$ was $56 \%$, twenty nine percent of them were able to identify the World Health Organization (WHO) five moments of $\mathrm{HH}$ while only $17 \%$ practiced $\mathrm{HH}$ during observation ${ }^{14}$. However, forgetfulness, negligence and unavailability of sanitizers were the top three reasons for not disinfecting their hands ${ }^{17}$.

Medical students play an essential role in delivering healthcare to the patients. Therefore, it is crucially important to evaluate their knowledge and practice of $\mathrm{HH}^{6,10}$. Moreover, adherence to $\mathrm{HH}$ between medical student contribute to the reduction of HAIs rates ${ }^{2}$. Hence, constant efforts are established to recognize efficient and sustainable methods to address this problem ${ }^{1,12}$. One of these efforts was introduced by the WHO which was the (My five Moments for Hand Hygiene) approach. These five moments include the following: before touching a patient, the moment before performing an aseptic or clean procedure, following exposure to body fluids, after touching a patient, and after touching any of the patient surroundings 1,12 .

Even though frequent reports about the knowledge of HH among medical students in different countries have been found, Saudi Arabia has greatly fallen behind in this field ${ }^{6}$. Such studies are of great value as students fill a large number in healthcare facilities during their training phase and may contribute to the transmission of infections. Moreover, their training attitude towards $\mathrm{HH}$ will reflect on their future infection control practice ${ }^{16}$.

Accordingly, we aimed for evaluating hand hygiene knowledge and practice between first and last clinical years; (i.e. fourth and sixth year medical students, respectively) at King Abdulaziz University, Jeddah, Saudi Arabia.

\section{Materials and Methods:-}

Study design

A questionnaire based, cross-sectional study with a single blinded (covert) observation.

\section{Study period and duration:-}

The study was undertaken from April to December 2015; a period of eight months.

\section{Study setting and population:-}

The study was conducted at King Abdulaziz University Hospital (KAUH) in Jeddah, Saudi Arabia on 261 medical students from the fourth and sixth year. Fourth year medical students were chosen because it is the first clinical year being exposed to patients, and they have already completed their Microbiology course which included a lecture about HH. On the other hand, sixth year medical students are in their graduation year and are expected to practice $\mathrm{HH}$ effectively, also they took a practical session about $\mathrm{HH}$.

\section{Data collection process:-}

The study is comprised of two parts: covert observation and a questionnaire which was self-administered. First, observation was among 22 groups, each group consisted of about ten to 13 students. It was done by a single student from each group who wasn't included in the study and was willingly accepting to observe his/her fellow group members, and was trained to blindly observe the practice of $\mathrm{HH}$ in his group members according to the WHO five Moments of Hand hygiene.

On the observation form, observers recorded where observation took place, the gender and clinical year of students observed, number of students in each ward, facilities available and the opportunities of hand hygiene the students encountered. Then a questionnaire was filled out by each student that has been observed earlier after obtaining an informed written consent. The questionnaire was designed according to WHO five moments of hand hygiene to estimate their knowledge using a five-points Likert scale ranging from strongly agree to strongly disagree. It also explored the participant's demographic data with no identifying data recorded other than the clinical year and the gender of the student to protect the student's confidentiality.

\section{Statistical analysis:-}


SPSS version 21 was used for all data entry and analysis. The association was tested by using Pearson's Chi-Square. Any p value less than 0.05 was considered statistically significant.

\section{Ethical consideration:-}

The ethical approval for the study was obtained from the Ministry of Higher Education, King Abdulaziz University, Faculty of Medicine Research Ethics Committee.

\section{Results:-}

Most of the medical students included in the study were fourth year students accounting as 157 students (60\%), and 104 students were sixth year (40\%). Male students were $162(62 \%)$ and female students were $99(38 \%)$.

HH practice among fourth and sixth year medical students

Practice among sixth year medical students was found to be better than fourth year medical students $(p=0.004)$. The number of sixth year medical students who applied HH was 60 (58\%), while 44 (42\%) did not apply HH. On the other hand, fourth year medical students who applied HH were 61 (39\%), while 96 (61\%) did not apply HH. As shown in (Figure 1)

HH knowledge among fourth and sixth year medical students

The knowledge between fourth and sixth year medical students was similar and found to be high $(\mathrm{p}=0.5)$, ranging between strongly agree and agree in the questionnaire. Among sixth year students, 76 students (75\%) chose strongly agree and 25 students $(25 \%)$ chose agree. On the other hand, fourth year students who chose strongly agree were $120(80 \%)$ and $30(20 \%)$ chose agree. (Figure 2$)$

The influence of role models' practice of $\mathrm{HH}$ on fourth and sixth year medical students

The performance of $\mathrm{HH}$ by the role models (i.e., physicians) strongly influenced the students' HH practice ( $p$ $<0.001)$. When the role model applied HH, 64 students (40\%) applied HH as well. Meanwhile, when they did not apply HH 50 students (31\%) did the same. (Figure 3)

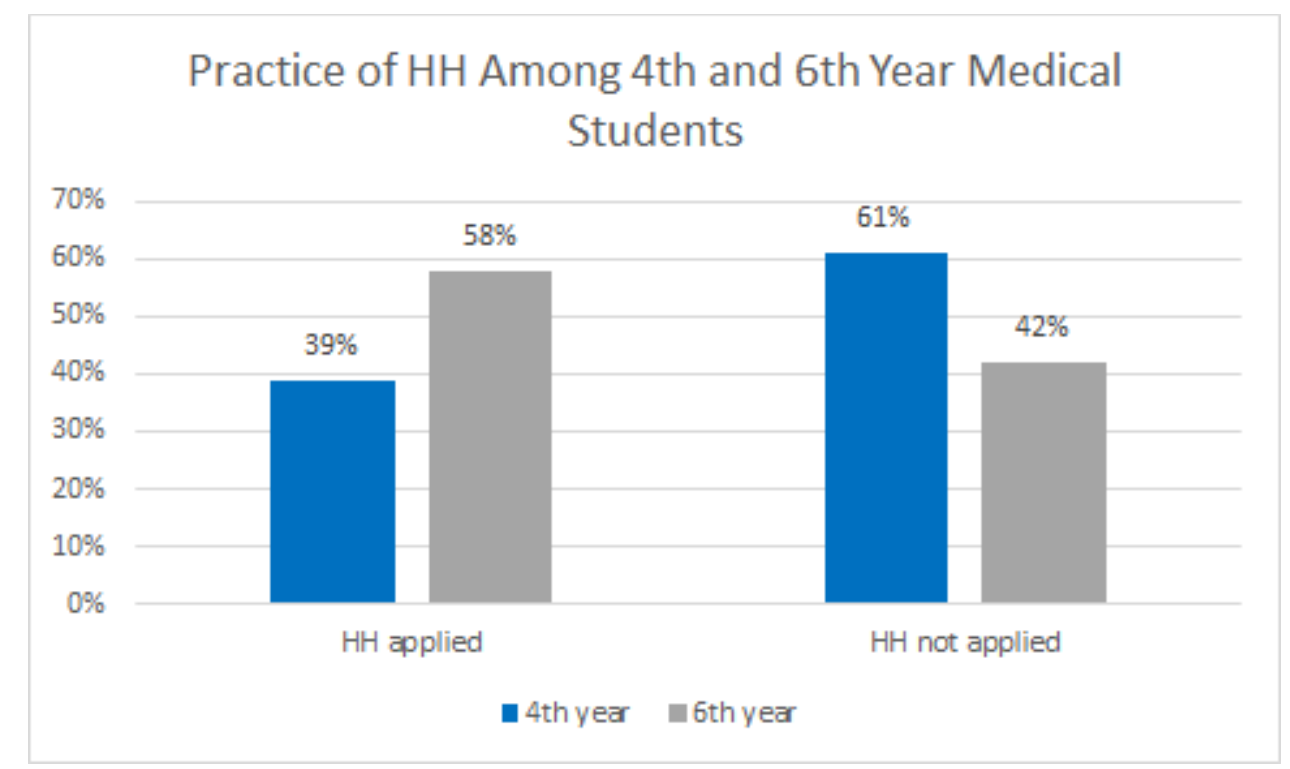

Figure 1 


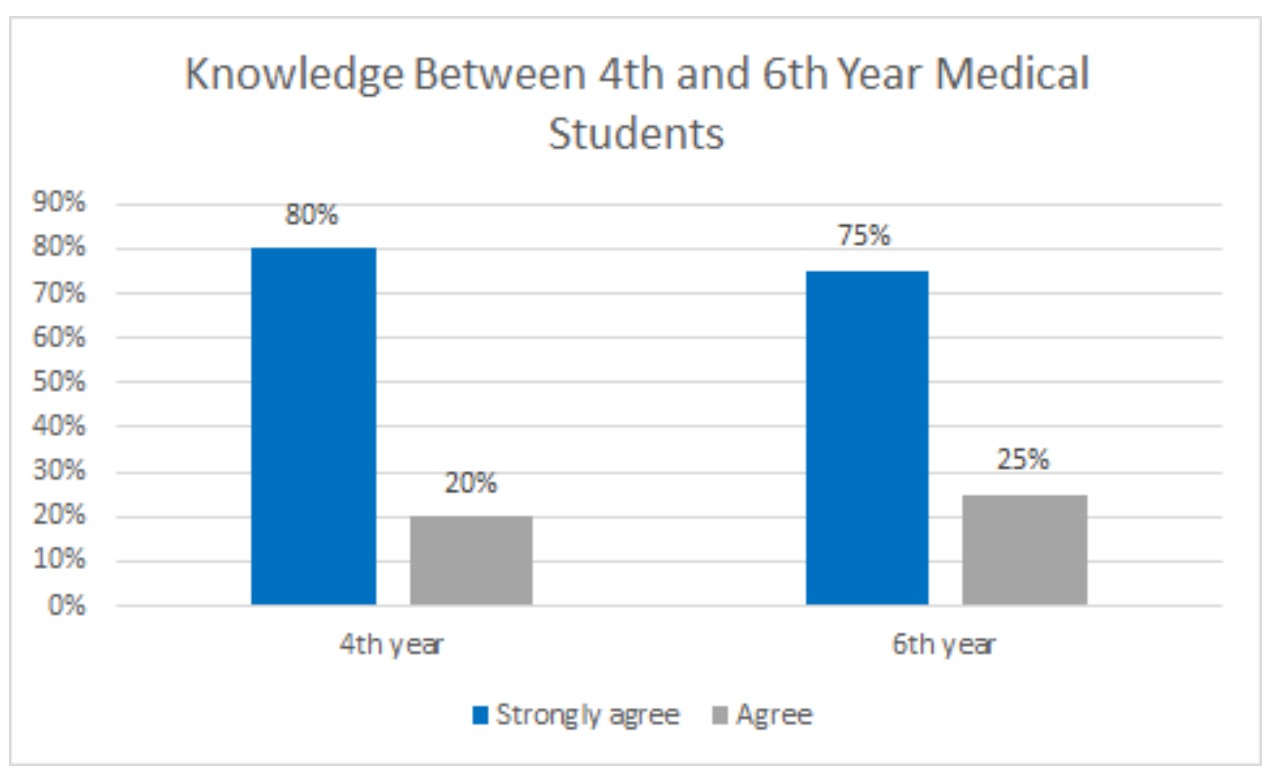

Figure 2

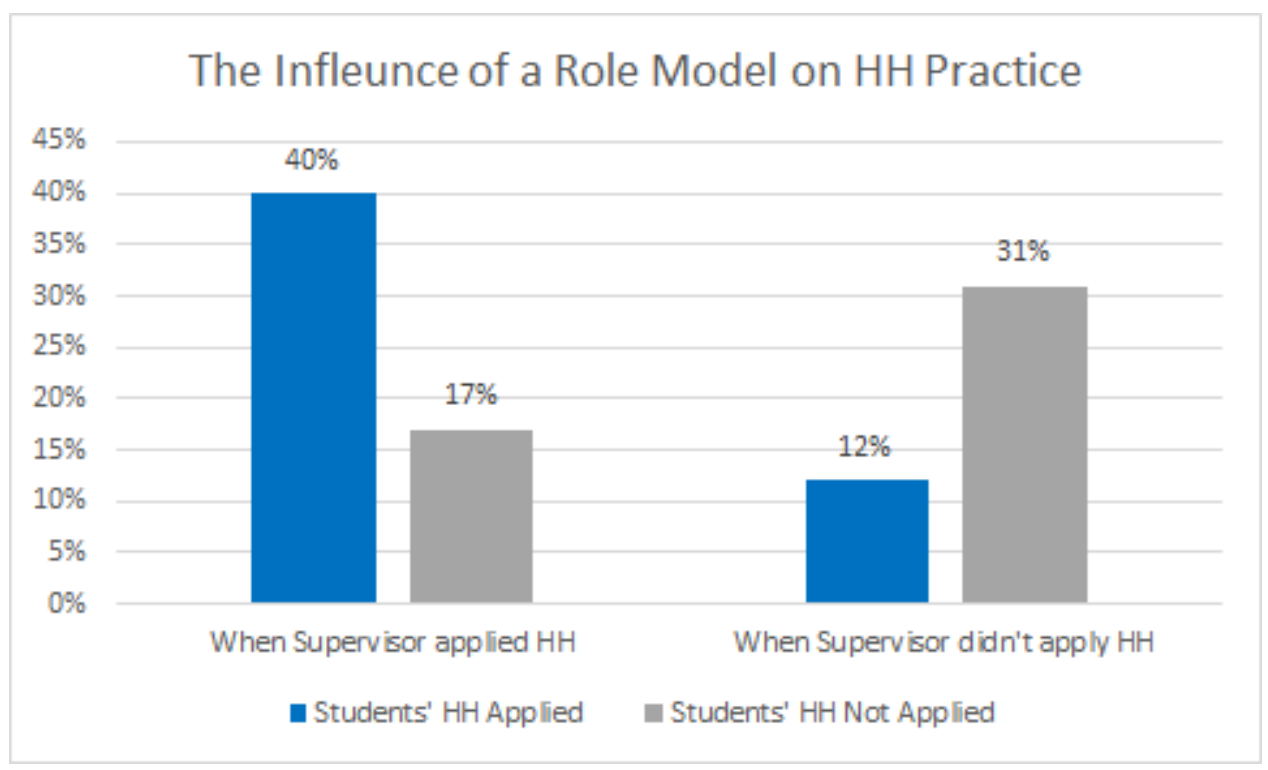

Figure 3

\section{Discussion:-}

Hand hygiene is an effortless measure to prevent hospital acquired infections. 'Clean Care is Safer Care' Was the earliest international challenge of the Union for Patient Safety of WHO ${ }^{18}$. Studies about HH knowledge and compliance have been deficient in the western province of Saudi Arabia. This study surveyed the knowledge about $\mathrm{HH}$ among fourth and sixth year medical students as well as their practice. Consistent with other studies, the knowledge level was found to be high among sixth and fourth year medical students ${ }^{13,19}$. As Van de Mortel et al. and Polacco et al, described that the many of medical students recognized the correct indications of $\mathrm{HH}$ and its importance ${ }^{1,20}$. The fact that both clinical years had similar results in terms of high knowledge could be attributed to them sharing the same curriculum which teaches the WHO five moments of HH in the preclinical year. A study accredited the high knowledge of their students to the fact that they have community medicine in their curriculum. In addition, there are many posters distributed among KAUH wards, hallways and patients' rooms which help in promoting the five moments of $\mathrm{HH}$. On the contrary, some studies showed that students had below average HH knowledge particularly regarding the WHO five moments of $\mathrm{HH}^{16}$. 
Regarding the compliance of $\mathrm{HH}$, students from sixth year were found to have better compliance than fourth year students. Which might be attributed to their better knowledge of infectious diseases including HAIs. Although, A study conducted on medical students and residents showed that the students were those who had the higher HH compliance ${ }^{21}$. Another study reported that $\mathrm{HH}$ compliance declined among physicians as they reached higher levels in the medical hierarchy, in the opinion of medical students ${ }^{22}$.

Other than knowledge which could be a factor influencing compliance, sixth year students are given a practical session that teaches the correct $\mathrm{HH}$ practice as part of their curriculum in the patient safety rotation. A study done in Germany by Stock et al. supports the importance of practical sessions in which their study showed that $\mathrm{HH}$ compliance was significantly improved after attending hands-on training sessions in the skills lab ${ }^{23}$. Sessions and activities for $\mathrm{HH}$ may also increase medical students' knowledge rather than just their compliance to $\mathrm{HH}$ as suggested by Kaur et al. ${ }^{24}$.

A study in Nepal described that the lack of role models for infection control and formal training of $\mathrm{HH}$ could be considered as possible barriers to apply correct $\mathrm{HH}$ measures ${ }^{25}$.

Accordingly, throughout our study, it was found that students' practice of HH was affected by the performance of their role models which was comparable to the findings of Hamadah et al. in which they stated that students lack proper HH practice in the absence of living examples (physicians, residents) ${ }^{6}$. The majority of students in a study conducted in Australia, felt that acting as role models for $\mathrm{HH}$ in regards to other healthcare workers was considered as their responsibility ${ }^{27}$. In addition, The practice of $\mathrm{HH}$ among superior mentors was described as the the main predictor of $\mathrm{HH}$ compliance among students ${ }^{26}$. Polacco et al. suggested that students and interns are less probably to perform $\mathrm{HH}$ if it wasn't applied by their faculty physicians ${ }^{1}$.

\section{Limitations:-}

Throughout the study, the significant limitation we faced was that the sample size of requirement was not fulfilled since several students were not willing to participate. Additionally, data collectors were not included in the study accounting as twenty-six students.

\section{Conclusion:-}

Strict protocols are needed for implementation of HH practice among students. As this study aimed for evaluating the knowledge about $\mathrm{HH}$ as well as the practice among fourth and sixth year medical students and showed that sixth year students have better practice of HH than fourth year students. Meanwhile, their knowledge level was found to be similar.

\section{Recommendations:-}

Several strategies can be done to improve the HH knowledge and practice on the behalf of medical students. Frequent training sessions need to be implemented earlier in the curriculum to enhance HH practice of medical students to have a better HH practice and therefore better infection control. Furthermore, these sessions should be associated with continuous assessment of the students' performance. It is also recommended that further research need to be undertaken to assess the proper technique and effectiveness of $\mathrm{HH}$. Not to mention evaluating $\mathrm{HH}$ on other healthcare providers.

\section{Acknowledgements:-}

The authors gratefully acknowledge Dr.Hanan Al Kadi and all who assisted in the statistical analysis including Ms.Nibal Abu Samaan and are thankful to all the data collectors, especially Rawan Jaha, Fatima Alzayer and Nawaf Bin Dhabab. 


\section{References:-}

1. Polacco MA, Shinkunas L, Perencevich EN, Kaldjian LC, Reisinger HS. See one, do one, teach one: Hand hygiene attitudes among medical students, interns, and faculty. American journal of infection control. 2015 Feb $1 ; 43(2): 159-61$.

2. Herbert VG, Schlumm P, Kessler HH, Frings A. Knowledge of and adherence to hygiene guidelines among medical students in Austria. Interdisciplinary perspectives on infectious diseases. 2013 Apr 11;2013.

3. Limper HM, Barton G, McGinty M, Landon E, O'boyle C, Reddy S, Weber SG. Behavioral intention of physician trainees and medical students to practice hand hygiene. Infection Control \& Hospital Epidemiology. 2013 Oct 1;34(10):1102-5.

4. Thakker VS, Jadhav PR. Knowledge of hand hygiene in undergraduate medical, dental, and nursing students: A cross-sectional survey. Journal of family medicine and primary care. 2015 Oct;4(4):582.

5. Ariyarathne MH, Gunasekara TD, Weerasekara MM, Kottahachchi J, Kudavidanage BP, Fernando SS. Knowledge, attitudes and practices of hand hygiene among final year medical and nursing students at the University of Sri Jayewardenepura. Sri Lankan Journal of Infectious Diseases. 2013 Feb 6;3(1).

6. Hamadah R, Kharraz R, Alshanqity A, AlFawaz D, Eshaq AM, Abu-Zaid A. Hand Hygiene: Knowledge and Attitudes of Fourth-Year Clerkship Medical Students at Alfaisal University, College of Medicine, Riyadh, Saudi Arabia. Cureus. 2014 Dec;7(8):e310-.

7. Sallami ZA. Assessment of Hand Hygiene Attitude, Knowledge and Practice among Health Science Students in Aden University. Journal of Biosciences and Medicines. 2016 Sep 6;4(09):25.

8. Jagdish L, Naik TB, Gupta RK, Upadhya AK. Prevalence of hand hygiene awareness among medical and dental students in a teaching hospital. Hand. 2016;46(32):0-12.

9. Mausumi Basu et al., SJAMS September 2015; Assessment of Hand Hygiene Knowledge among Medical Students at a Teaching Hospital of India. 3(6C):2334-2339.

10. Senanayake NP, Navaratne V, Balasuriya A. Knowledge of Hand Hygiene among the Medical Students at the General Sir John Kotelawala Defence University. InKDU INTERNATIONAL RESEARCH CONFERENCE20142015 Feb (p. 10).

11. Bukhari SZ, Hussain WM, Banjar A, Almaimani WH, Karima TM, Fatani MI. Hand hygiene compliance rate among healthcare professionals. Saudi medical journal. 2011;32(5):515-9.

12. Nair SS, Hanumantappa R, Hiremath SG, Siraj MA, Raghunath P. Knowledge, attitude, and practice of hand hygiene among medical and nursing students at a tertiary health care centre in Raichur, India. ISRN preventive medicine. 2014 Feb 6;2014.

13. Joshi SC, Diwan V, Tamhankar AJ, Joshi R, Shah H, Sharma M, et al. Qualitative study on perceptions of hand hygiene among hospital staff in a rural teaching hospital in India. Journal of Hospital Infection. 2012 Apr 30;80(4):340-4.

14. Al Kadi A, Salati SA. Hand hygiene practices among medical students. Interdisciplinary perspectives on infectious diseases. 2012 Sep 16;2012.

15. Scheithauer S, Haefner H, Schwanz T, Lopez-Gonzalez L, Bank C, Schulze-Röbbecke R, et al. Hand hygiene in medical students: performance, education and knowledge. International journal of hygiene and environmental health. 2012 Sep 30;215(5):536-9.

16. Mohammed A, Aliyu I. Hand-washing practices and techniques among clinical students of Bayero University Kano, Nigeria. Sudan Medical Monitor. 2015 Apr 1;10(2):51.

17. Kalata NL, Kamange L, Muula AS. Adherence to hand hygiene protocol by clinicians and medical students at Queen Elizabeth Central Hospital, Blantyre-Malawi. Malawi Medical Journal. 2013;25(2):50-2.

18. Lucas NC, Hume CG, Al-Chanati A, Diprose W, Roberts S, Freeman J, et al. Student-led intervention to inNOvate hand hygiene practice in Auckland Region's medical students (the No HHARMS study). The New Zealand medical journal. 2017 Jan 13;130(1448):54.

19. Sulaiha SA, Ling WY, Chin LJ, Eow WL, MuhamadFaiz S, Tan NC. The lack of effective hand washing practice despite high level of knowledge and awareness in medical students of clinical years. IeJSME. 2010;4(2):18-26.

20. Van de Mortel TF. Development of a questionnaire to assess health care students' hand hygiene knowledge, beliefs and practices. Australian Journal of Advanced Nursing. 2009;26(3):9-16.

21. Barroso V, Caceres W, Loftus P, Evans KH, Shieh L. Hand hygiene of medical students and resident physicians: predictors of attitudes and behaviour. Postgraduate medical journal. 2016 Feb 24:postgradmedj2015.

22. Graf K, Chaberny IF, Vonberg RP. Beliefs about hand hygiene: a survey in medical students in their first clinical year. American journal of infection control. 2011 Dec 31;39(10):885-8. 
23. Stock S, Tebest R, Westermann K, Samel C, Strohbücker B, Stosch C, et al. Implementation of an innovative hands-on training to improve adherence to hygiene rules: A feasibility Study. Nurse education today. 2016 Jan 31;36:407-11.

24. Kaur R, Razee H, Seale H. Development and appraisal of a hand hygiene teaching approach for medical students: a qualitative study. Journal of Infection Prevention. 2016 Jul;17(4):162-8.

25. Paudel IS, Ghosh V, Adhikari P. Knowledge, Attitude and Practice of nursing students on Hospital Acquired Infections in Western region of Nepal. Journal of College of Medical Sciences-Nepal. 2016 Nov 23;12(3):1037.

26. Snow M, White GL, Alder SC, Stanford JB. Mentor's hand hygiene practices influence student's hand hygiene rates. American journal of infection control. $2006 \mathrm{Feb} 28 ; 34(1): 18-24$.

27. Kaur R, Razee H, Seale H. Setting the right foundations: improving the approach used to teach concepts of hand hygiene to medical students. Journal of Hospital Infection. 2017 Apr 30;95(4):355-8. 